In der Rubrik „Literatur kompakt" werden die wichtigsten Originalarbeiten aus der internationalen Fachliteratur referiert.

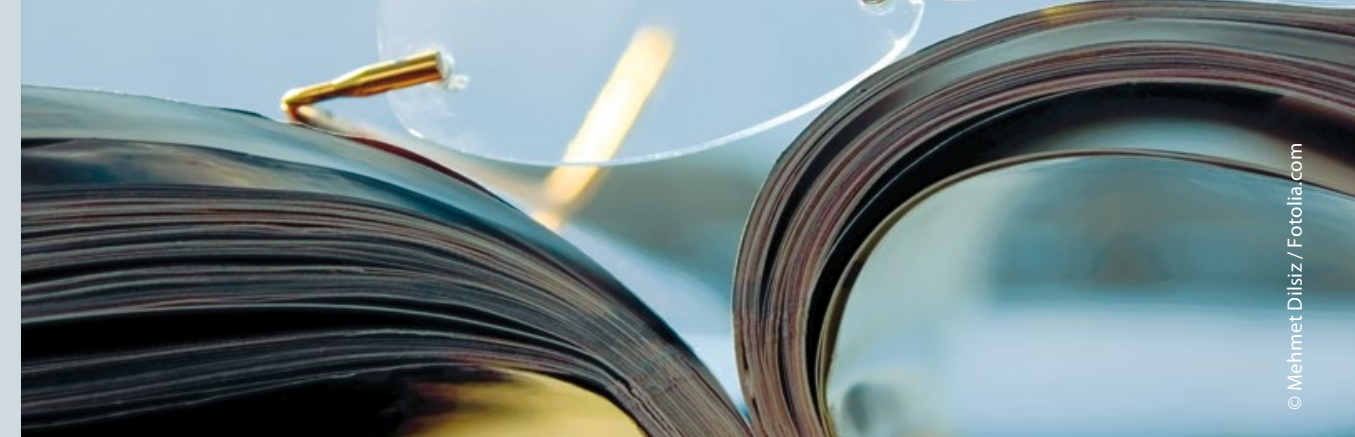

\section{Bienengift-SIT: individualisierte Präparateauswahl}

\author{
Bienengift enthält eine Vielzahl von Majorallergenen. Das dürfte auch \\ Auswirkungen auf die Präparateauswahl für die spezifische Immuntherapie \\ haben. Eine Antikörperstudie untersuchte jetzt die Majorallergen- \\ Zusammensetzung verschiedener SIT-Präparate.
}

ange Zeit galt Api m 1 als das ent— scheidende Bienengift-Majorallergen. Erst in neuerer Zeit wurden weitere Proteine mit nicht zu vernachlässigenden Sensibilisierungsraten entdeckt: Api m 2, Api m 3, Api m 5 und Api m 10 mit sIgEReaktivitäten von $47,9-52,2 \%, 49,6-$ $50,0 \%, 58,3-61,7 \%$ und $61,8-72,2 \%$. Verglichen mit Api $\mathrm{m} 1$ und dem Minorallergen Api m 4 liegen diese Allergene allerdings nur in geringen Konzentrationen im Bienengift vor - und können bei der Aufbereitung des Gifts zu therapeutischen Zwecken leicht verloren gehen. Dies legen erste Untersuchungen nahe.
Möglicherweise ist eine zu geringe Konzentration einiger Majorallergene in SIT-Präparaten auch eine Erklärung für die bei Bienengiftallergikern im Vergleich zu Wespengiftallergikern geringeren Erfolgsraten einer Hyposensibilisierung - Untersuchungen zeigen nämlich recht heterogene Sensibilisierungsmuster bei Bienengiftallergien.

In einer neuen Studie sollten die ersten Erkenntnisse zum Majorallergengehalt von handelsüblichen BienengiftSIT-Präparaten erneut überprüft werden. Eingesetzt wurden dabei hoch spezifische und hoch sensitive Antikörper zum

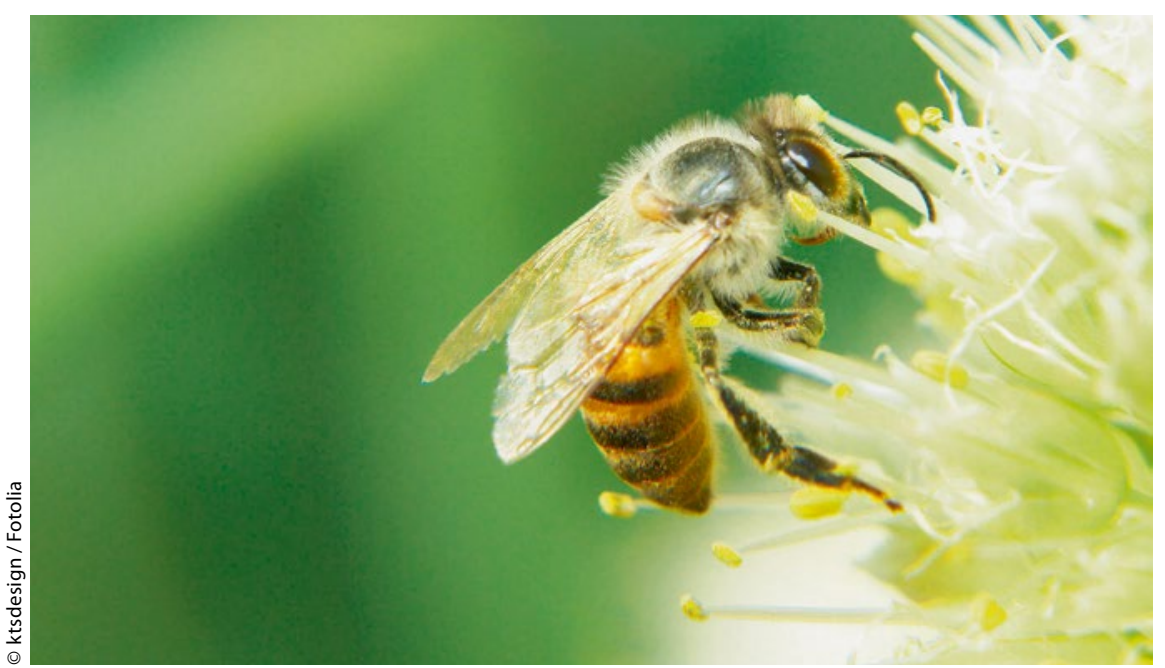

Nicht alle Bienengiftallergene finden sich auch in SIT-Präparaten.
Nachweis der Majorallergene Api m 2, Api m 3, Api $m 5$ und Api m 10. Auch hier bestätigte sich: Die einzelnen SITPräparate unterscheiden sich im Majorallergengehalt, bei einigen Produkten sind vor allem Api $\mathrm{m} 3$ und Api m 10 unterrepräsentiert oder nicht nachweisbar. Begründet werden kann dieses Resultat mit den unterschiedlichen Aufbereitungsprozeduren des Rohgifts durch die einzelnen Hersteller und geografischen oder saisonalen Schwankungen des natürlichen Rohstoffs Bienengift, diskutieren die Autoren.

Vermutlich haben diese Befunde auch klinische Relevanz, schreiben die Autoren weiter. Eine kürzlich publizierte Studie ergab eine Korrelation zwischen einem unzureichenden Erfolg einer Bienengift-SIT und einer dominanten Sensibilisierung der Patienten gegen das Majorallergen Api m 10. In diesen Fällen sollte ein SIT-Präparat mit ausreichendem Api-m-10-Gehalt eingesetzt und umgekehrt Patienten mit nicht nachweisbarer Api-m-10-Sensibilisierung ein Api-m-10-armes Produkt angeboten werden. Prospektive klinische Studien zu dieser Fragestellung stehen allerdings noch aus.

Fazit: Bienengift-SIT-Präparate zeigen einen weitgehend vergleichbaren Gehalt des Majorallergens Api m 1, unterscheiden sich aber in der Zusammensetzung von weiteren wichtigen Allergenen wie Api m 3 und Api m 10.

\section{Dr. Barbara Kreutzkamp}

Blank $\mathrm{S}$ et al. Component-resolved evaluation of the content of major allergens in therapeutic extracts for specific immunotherapy of honeybee venom allergy. Human Vaccin Immunother 2017; 11: 1-8 